\title{
Building a Bridge Between Research and Practice- The Importance of the Practical Application
}

A strength of many of the papers published in the International Journal of Sports Physiology and Performance (IJSPP) is that the findings have clear translation or impact on practice. Practical application of research is central to the journal's mission and also to the work of our many contributors. Indeed, when you browse a table of contents of any issue of IJSPP, it is obvious that many studies have a strong applied focus. Collectively, these studies contribute to improving professional practice, by providing an improved evidence base that can be used to inform decisions around athlete preparation and competition. For these reasons, the "Practical Applications" section is critical to papers published in IJSPP. In this editorial, I discuss the importance of the "Practical Applications" section, identify common difficulties in providing authentic recommendations, and provide advice on how to overcome these problems.

IJSPP's author guidelines state that the "Practical Applications" section, positioned at the end of manuscripts, should summarize how the findings could be useful for coaches and athletes and/or other researchers in sport physiology and performance. The study's limitations and generalizability should also be addressed and recommendations made for future research, where necessary. This section is critical in assisting readers to comprehend how findings may be applied in practice. These translational statements can also be important for authors, acting as a source of credibility with our end users, which may lead to additional rewards (ie, increased recognition, research funding, consultancies, etc). However, because of these benefits, it may be tempting to overinterpret or oversimplify the research results and/or provide catchy statements that may not be directly supported by the findings. We caution against this as it will ultimately undermine the credibility of our work.

The perceived value of research findings often depends on how well they can be translated into practice to improve outcomes. Even with strong applied research designs, it can be a challenge to translate our findings into authentic practical advice for athletes and practitioners. Whether the outcome is improved performance, the optimization of a training program, more effective monitoring, injury reduction, improved rehabilitation and return to sport, or improving skills or tactics, the end users often require guidance on how to put rather complex findings into practice. However, translating study findings from controlled research settings into the much less controlled environment of high-performance sport can be challenging. Therefore, clear practical advice on the use of a study's findings is essential. Similarly, acknowledging when there are no clear practical applications is also important.

As many coaches and athletes also have an eagerness for new knowledge or advice that may enable them to improve their performance, it is logical that they look to the practical application advice in papers to inform their decision making. Indeed, these statements are often used as the take-home messages or as social media summaries. Clearly, interpreting research based on limited information (ie, without reading the paper) is risky, but even more so when initial practical statements are not supported by the data. Therefore, authors should be careful to ensure that the statements are well supported by their findings and that any concerns about the generalizability of the findings are addressed. Obvious examples of these risks occur when making general statements about the usefulness of tools for predicting talent, injury, or performance changes based on case studies or limited data. We should also take care in making practical recommendations from descriptive studies, as the design of these often prevents formation of clear recommendations.

It is the responsibility of researchers to provide recommendations that are supported by evidence and interpreted in the context of each study's limitations. It is also the responsibility of reviewers to check the "Practical Applications" section carefully, to ensure that false promises are not being made. If we fail to achieve this, false promises will be made, and these will ultimately undermine trust in the field.

As an associate editor for IJSPP, I have the privileged position of gaining insight into expert reviewer feedback on many articles, and that provides insights into what to avoid. Some of the common criticisms are that the "Practical Applications" sections often simply restate the results, recommendations are not directly supported by the data, and they can lack authentic practical relevance for the end user. Others are criticized for their theoretical shortcomings and lack of direct empirical support. These criticisms should also be considered when developing future practical advice for end users.

We advise that authors check the relevance of their practical recommendations with experienced coaches, athletes, and applied scientists. Such feedback from different points of view can be of great benefit and may be a good place to start if you have doubt. It is also prudent to clearly note when the findings do not directly apply to practice. In such cases, the advice provided may be to researchers about specific future work that needs to be done before practical translation might be possible. In any case, it is responsible to set realistic expectations about the generalizability of the findings-it is often practically relevant to state what our findings do not show as much as what they do.

With a rapidly increasing number of published studies and contrasting messages in research (and social media), it can be challenging to provide clear practical advice that is based on evidence. The intent of this editorial was to draw your attention to the importance of developing useful practical applications of our work and the responsibility we have in articulating these clearly. Ultimately, the responsibility is on the readers to understand the uncertainty in any research findings, but this is much easier to achieve if we as authors provide clear and relevant practical advice.

Aaron J. Coutts, IJSPP Associate Editor, University of Technology Sydney (UTS), Australia 